\title{
Spontaneous Umbilical Hernia Rupture Associated With Omentum Evisceration in a Patient With Advanced Hepatic Cirrhosis and Refractory Ascites
}

Abdulqader M. Albeladi ${ }^{1}$, Ahmad M. Odeh ${ }^{1}$, Aminah H. AlAli ${ }^{2}$, Abdullah M. Alkhars ${ }^{3}$, Adeeb M. Buhlaigah $^{4}$, Hussain A. Alghadeer ${ }^{5}$, Mohammed J. Almosbeh ${ }^{2}$, Mohmmed T. AlAbbad ${ }^{2}$, Mohammad S. AlGhadeer $^{2}$

1. Laparoscopic Surgery, Prince Saud Bin Jalawi Hospital, Al-Ahsa, SAU 2. General Surgery, Prince Saud Bin Jalawi Hospital, Al-Ahsa, SAU 3. Orthopaedics, King Fahad Hospital, Al-Ahsa, SAU 4. Orthopaedics, King Faisal University, Al-Ahsa, SAU 5. Pediatrics, Maternity and Children Hospital, Al-Ahsa, SAU

Corresponding author: Hussain A. Alghadeer, hu.alghadeer@gmail.com

\section{Abstract}

Flood syndrome is a spontaneous rupture of an umbilical hernia. It has a high mortality and morbidity and presents many challenges in medical versus surgical management. We present a case of a 23 -year-old Yamani woman with complicated umbilical hernia, newly diagnosed hepatitis B infection, and decompensated liver cirrhosis with ascites (Child-Pugh grade B). The patient was undergoing multiple abdominal ascitic tapping that eventually ruptured with an omentum evisceration, causing Flood syndrome. An urgent umbilical hernioplasty with mesh in a sublay technique was conducted.

Categories: Gastroenterology, General Surgery

Keywords: flood syndrome, umbilical hernia, liver cirrhosis, refractory ascites, omentum evisceration

\section{Introduction}

Ascites is a common complication of cirrhosis and indicates a new phase of hepatic decompensation in the progression of the cirrhotic process [1]. The mechanism that leads to the development of ascites in cirrhosis is multifactorial. Severe portal hypertension and hepatic insufficiency are the initial factors. A combination of increased intra-abdominal pressure from ascites and poor nutritional status then leads to the weakening of the anterior abdominal wall muscles, creating a defect [2]. Flood syndrome is described as a sudden gush of fluid from spontaneous umbilical hernia rupture. It is a life-threatening complication of chronic ascites and end-stage liver disease [3]. Estimated mortality rate is $30 \%$. Up to $20 \%$ of patients with ascites in a hepatic cirrhosis setting develop umbilical hernias [2,3]. An umbilical hernia in these patients has a tendency to enlarge rapidly and to cause complications. Subsequently, it can rupture [4].

Review began 06/18/2021 Review ended 06/23/2021 Published 06/29/2021

() Copyright 2021

Albeladi et al. This is an open access article distributed under the terms of the Creative Commons Attribution License CC-BY 4.0., which permits unrestricted use, distribution, and reproduction in any medium, provided the original author and source are credited.
This case report presents the challenge in management of spontaneous rupture of an umbilical hernia which is a rare complication with high mortality rates and stresses the challenge of treatment that falls in the area between medical and surgical management.

\section{Case Presentation}

A 23-year-old Yamani woman presented to our emergency department with a case of abdominal pain and distension. Examination and investigations found an uncomplicated umbilical hernia, liver cirrhosis and ascites. The patient was admitted by the medical team, who investigated the patient for two weeks, eventually diagnosing a case of hepatitis B infection and decompensated liver cirrhosis (Child-Pugh grade B). After two weeks under medical care and multiple ascitic tapping, the patient responded with a sudden gush of clear fluid and spontaneous evisceration of the omentum from the umbilical hernia (Figure 1). The patient was referred from the medical team as a case of spontaneous umbilical hernia rupture and leaking ascitic fluid with omentum evisceration. 


\section{Cureus}

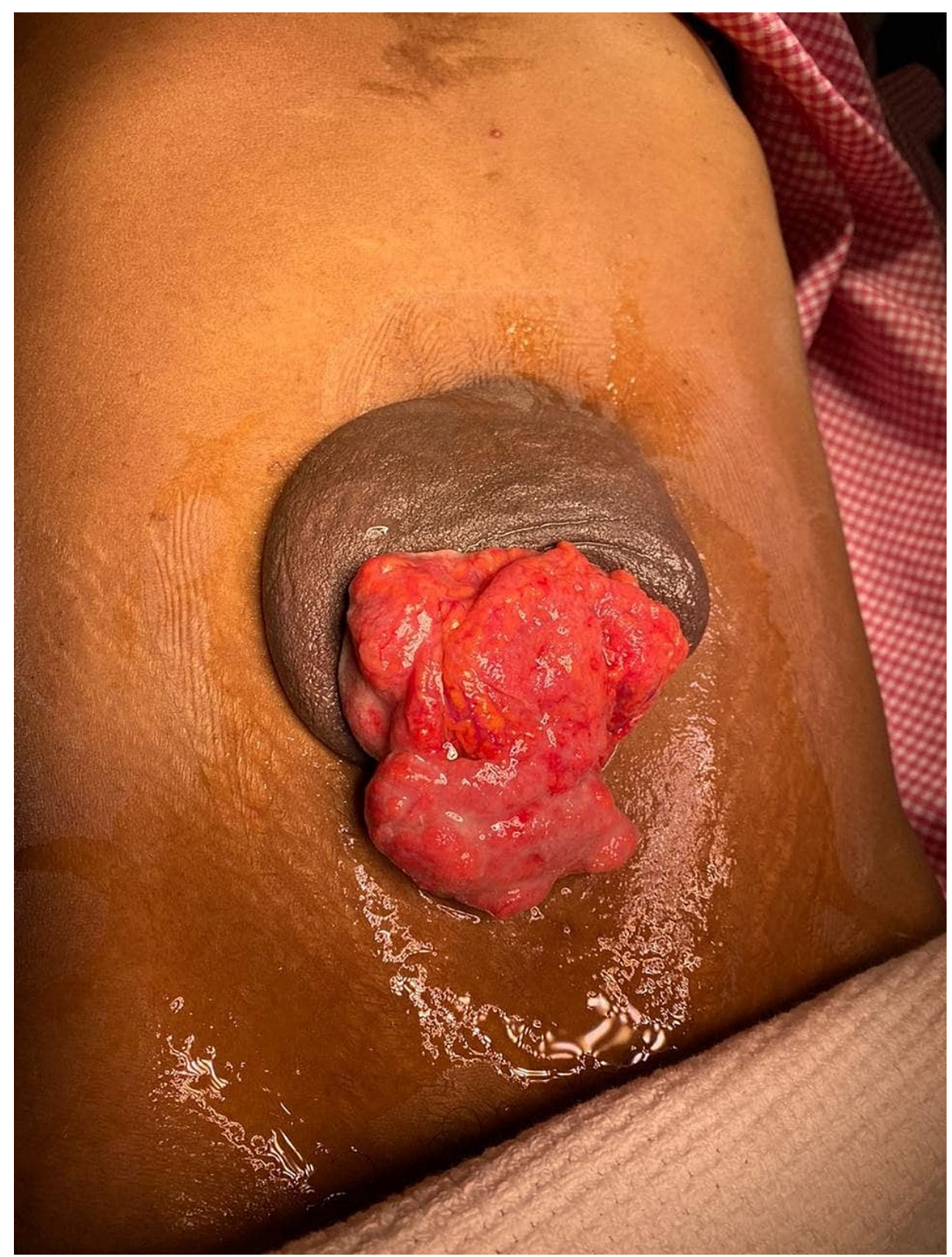

\section{FIGURE 1: Spontaneous rupture of an umbilical hernia associated with omentum evisceration.}

An urgent laparotomy was performed after preparation of the skin with an antiseptic solution. Excision of the umbilicus and eviscerated omentum was done. Aspiration of all ascitic fluid and samples were sent for cytology, biochemical, and microbiology testing. All abdominal viscera were examined, but no evidence of the recanalization of the umbilical vein was found.

After placement of an abdominal drain, a blunt dissection was done between the posterior rectus sheath and the rectus abdominus to facilitate placement of the polypropylene mesh in a sublay position. The posterior rectus sheath was closed using $1 / 0$ non-absorbable polypropylene stitch. The mesh was placed over the posterior rectus sheath and below the rectus abdominus. The anterior rectus sheath was closed using 1/10 polypropylene stitch, and the skin was closed using clips.

Postoperatively, the patient stayed in the ICU for five days and was then moved to a regular ward. She received prophylactic broad spectrum antibiotics and albumin. Her abdominal drain output ranged from 1000-2000 ml clear ascitic fluid per day for one week, then decreased gradually over the next week to around $30 \mathrm{ml}$. The drain was removed, and the patient was discharged home. During follow-up in the outpatient clinic after two weeks, the patient was doing well with no wound complications. Consequently, the patient was referred to a tertiary center for further management. 


\section{Discussion}

The spontaneous rupture of an umbilical hernia, known as Flood syndrome, is a rare occurrence that can be accompanied by significant morbidity and mortality. It was first described in the literature in $1901[2,3]$. Complications of umbilical hernia rupture include an incarcerated bowel, spontaneous paracentesis and evisceration, hypotension, peritonitis, development of cellulitis, and sepsis [5]. Flood syndrome has high mortality and morbidity and presents many challenges in medical versus surgical management. The prevention of an umbilical hernia rupture depends on the optimal management of the underlying ascites in cirrhotic patients.

Conservative management includes the use of diuretics (furosemide and spironolactone), regular paracentesis, and non-steroidal inflammatory drugs with dietary salt and fluid restrictions [2]. Due to the difficulties Flood syndrome presents, surgical management is not a well-established procedure and is associated with a mortality rate of up to $30 \%$, especially in patients undergoing emergent hernia repair. In cases of failed conservative management, the surgical options include umbilical herniorrhaphy, peritoneovenous shunting (PVS), transjugular intrahepatic portosystemic shunting (TIPS), and concomitant portal venous decompression $[2,6]$. However, postoperative mortality can be as high as $60 \%$ to $80 \%$ and morbidity up to $71 \%$. Immediate surgical intervention decreases the mortality range to 6-20\% [5]. Moreover, the postoperative control of ascites is still difficult concerning repair and the prevention of complications.

Elective herniorrhaphy is well recommended in cirrhotic patients with well-controlled ascites and no comorbidities [7]. In cases of a complicated hernia or uncontrolled ascites, however, the procedure is usually associated with postoperative complications [7]. However, urgent umbilical herniorrhaphy without mesh or primary closure is the preferred intervention in cirrhotic patients presenting an umbilical hernia rupture since it has been shown to reduce the mortality rate to 6-20\% [2]. Our patient was treated with urgent surgical repair using mesh; some studies have reported instances of reduced hernia recurrence in patients who underwent mesh umbilical herniorrhaphy $[8,9]$. In our case, the patient was young and clinically stable with no signs of infection; hence, we decided to repair the hernia using the sublay technique because it has a lower rate of infection and recurrence $[8,9]$. The sublay technique can be used in elective repair and also in emergency situations [10,11]. Our patient, treated with an intraperitoneal drain, prophylactic broad spectrum antibiotics, albumin, and regular dressing, showed an improved abdominal distention and was discharged on day 14 in good condition. Finally, the postoperative control of ascites is mandatory to prevent recurrence and further complications.

\section{Conclusions}

Flood syndrome is a spontaneous rupture of the umbilical hernia. It has a high mortality and morbidity and presents many challenges in medical versus surgical management. Elective hernioplasty in non-complicated cases is well recommended in cirrhotic patients with well-controlled ascites and no comorbidities. In complicated situations, urgent umbilical herniorrhaphy or primary closure is the preferred intervention. However, either in elective or emergency situations, mesh can be used with different techniques.

\section{Additional Information \\ Disclosures}

Human subjects: Consent was obtained or waived by all participants in this study. Conflicts of interest: In compliance with the ICMJE uniform disclosure form, all authors declare the following: Payment/services info: All authors have declared that no financial support was received from any organization for the submitted work. Financial relationships: All authors have declared that they have no financial relationships at present or within the previous three years with any organizations that might have an interest in the submitted work. Other relationships: All authors have declared that there are no other relationships or activities that could appear to have influenced the submitted work.

\section{References}

1. Moore KP, Wong F, Gines P, et al.: The management of ascites in cirrhosis: report on the consensus conference of the International Ascites Club. Hepatology. 2003, 38:258-266. 10.1053/jhep.2003.50315

2. Chatzizacharias NA, Bradley JA, Harper S, et al.: Successful surgical management of ruptured umbilical hernias in cirrhotic patients. World J Gastroenterol. 2015, 21:3109-3113. 10.3748/wjg.v21.i10.3109

3. Drake CB, Arowojolu OA, Mitchell OJL, Liu S: FLOOD Syndrome: Not Your Average Paracentesis. Hospital Medicine, Las Vegas; 2017.

4. Ogu US, Valko J, Wilhelm J, Dy V: Spontaneous evisceration of bowel through an umbilical hernia in a patient with refractory ascites. J Surg Case Rep. 2013, 2013:rit073. 10.1093/jscr/rjt073

5. Liu GF, Srinivasan A, Mutnuri S, Yerramadha MR, Agraharkar M: Acute abdomen from umbilical hernia rupture to Flood syndrome: a case report and review of literature. J Med Cases. 2019, 10:309-311. 10.14740/jmc3375

6. Odom SR, Gupta A, Talmor D, Novack V, Sagy I, Evenson AR: Emergency hernia repair in cirrhotic patients with ascites. J Trauma Acute Care Surg. 2013, 75:404-409. 10.1097/TA.0b013e31829e2313

7. Choi SB, Hong KD, Lee JS, et al.: Management of umbilical hernia complicated with liver cirrhosis: an advocate of early and elective herniorrhaphy. Dig Liver Dis. 2011, 43:991-995. 10.1016/j.dld.2011.07.015 


\section{Cureus}

8. Eker $\mathrm{HH}$, van Ramshorst $\mathrm{GH}$, de Goede $\mathrm{B}$, et al.: A prospective study on elective umbilical hernia repair in patients with liver cirrhosis and ascites. Surgery. 2011, 150:542-546. 10.1016/j.surg.2011.02.026

9. McKay A, Dixon E, Bathe O, Sutherland F: Umbilical hernia repair in the presence of cirrhosis and ascites: results of a survey and review of the literature. Hernia. 2009, 13:461-468. 10.1007/s10029-009-0535-9

10. Hassan AM, Salama AF, Hamdy H, Elsebae MM, Abdelaziz AM, Elzayat WA: Outcome of sublay mesh repair in non-complicated umbilical hernia with liver cirrhosis and ascites. Int J Surg. 2014, 12:181-185. 10.1016/j.ijsu.2013.12.009

11. Yu BC, Chung M, Lee G: The repair of umbilical hernia in cirrhotic patients: 18 consecutive case series in a single institute. Ann Surg Treat Res. 2015, 89:87-91. 10.4174/astr.2015.89.2.87 